\title{
Solving the Matrix Nearness Problem in the Maximum Norm by Applying a Projection and Contraction Method
}

\author{
M. H. Xu' ${ }^{1}$ and H. Shao ${ }^{2}$ \\ ${ }^{1}$ School of Mathematics and Physics, Changzhou University, Jiangsu Province, \\ Changzhou 213164, China \\ ${ }^{2}$ Department of Mathematics, School of Sciences, China University of Mining and Technology, \\ Xuzhou 221116, China
}

Correspondence should be addressed to M. H. Xu, xuminghua@cczu.edu.cn

Received 11 April 2012; Accepted 17 June 2012

Academic Editor: Abdellah Bnouhachem

Copyright (C 2012 M. H. Xu and H. Shao. This is an open access article distributed under the Creative Commons Attribution License, which permits unrestricted use, distribution, and reproduction in any medium, provided the original work is properly cited.

Let $S$ be a closed convex set of matrices and $C$ be a given matrix. The matrix nearness problem considered in this paper is to find a matrix $X$ in the set $S$ at which $\max \left\{\left|x_{i j}-c_{i j}\right|\right\}$ reaches its minimum value. In order to solve the matrix nearness problem, the problem is reformulated to a min-max problem firstly, then the relationship between the min-max problem and a monotone linear variational inequality (LVI) is built. Since the matrix in the LVI problem has a special structure, a projection and contraction method is suggested to solve this LVI problem. Moreover, some implementing details of the method are presented in this paper. Finally, preliminary numerical results are reported, which show that this simple algorithm is promising for this matrix nearness problem.

\section{Introduction}

Let $C=\left(c_{i j}\right) \in R^{n \times n}$ be a given symmetric matrix and

$$
S_{\Lambda}^{n}=\left\{H \in R^{n \times n} \mid H^{T}=H, \lambda_{\min } I \leq H \leq \lambda_{\max } I\right\},
$$

where $\lambda_{\min }, \lambda_{\max }$ are given scalars and $\lambda_{\min }<\lambda_{\max }, I$ is the identity matrix, and $A \preceq B$ denotes that $B-A$ is a positive semidefinite matrix. It is clear that $S_{\Lambda}^{n}$ is a nonempty closed convex set. The problem considered in this paper is

$$
\min \left\{\|X-C\|_{\text {inf }} \mid X=\left(x_{i j}\right) \in S_{\Lambda}^{n}\right\},
$$


where

$$
\|X-C\|_{\text {inf }}=\max \left\{\left|x_{i j}-c_{i j}\right| \mid i=1, \ldots, n, j=1, \ldots, n\right\}
$$

Throughout the paper we assume that the solution set of problem (1.2) is nonempty.

Note that when $\lambda_{\min }=0$ and $\lambda_{\max }=+\infty$, the set $S_{\Lambda}^{n}$ reduces to the semidefinite cone

$$
S_{+}^{n}=\left\{H \in R^{n \times n} \mid H^{T}=H, H \geq 0\right\} .
$$

Using the terminology in interior point methods, $S_{+}^{n}$ is called semidefinite cone, and thus the related problem belongs to the class of semidefinite programming [1].

Problem (1.2) can be viewed as a type of matrix nearness problem, that is, the problem of finding a matrix that satisfies some property and is nearest to a given one. A survey on matrix nearness problems can be found in [2]. The matrix nearness problems have many applications especially in finance, statistics, and compressive sensing. For example, one application in statistics is to make adjustments to a symmetric matrix so that it is consistent with prior knowledge or assumptions, and is a valid covariance matrix [3-8]. Paper [9] discusses a new class of matrix nearness problems that measure approximation error using a directed distance measure called a Bregman divergence and proposes a framework for studying these problems, discusses some specific matrix nearness problems, and provides algorithms for solving them numerically.

Note that a different norm is used in this paper than in these published papers [3-7] and this makes the objective function of problem (1.2) be nonsmooth, and the problem (1.2) cannot be solved very easily. In the next section, we will find that the problem (1.2) can be converted into a linear variational inequality and thus can be solved effectively with the projection and contraction (PC) method which is extremely simple both in theoretical analysis and numerical implementations [10-15].

The paper is organized as follows. The relationship between the matrix nearness problem considered in this paper and a monotone linear variational inequality (LVI) is built in Section 2. In Section 3, some preliminaries on variational inequalities are summarized. The projection and contraction method for the LVI associated with the considered problem is suggested. In Section 4, the implementing details for applying the projection and contraction method to the matrix optimization problems are studied. Preliminary numerical results and some concluding remarks are reported in Sections 5 and 6, respectively.

\section{Reformulating the Problem to a Monotone LVI}

For any $d \in R^{m}$, we have

$$
\|d\|_{\infty}=\max _{\xi \in B_{1}} \xi^{T} d
$$

where $B_{1}=\left\{\xi \in R^{m} \mid\|\xi\|_{1} \leq 1\right\}$ and $\xi^{T} d$ is the Euclidean inner-product of $\xi$ and $d$. 
In order to simplify the following descriptions, let vec $(A)$ be a linear transformation which converts the matrix $A \in R^{l \times n}$ into a column vector in $R^{l n}$ obtained by stacking the columns of the matrix $A$ on top of one another, that is,

$$
\operatorname{vec}(A)=\left(a_{11}, \ldots, a_{l 1}, a_{12}, \ldots, a_{l 2}, \ldots, a_{1 n}, \ldots, a_{l n}\right)^{T},
$$

and let mat(vec $(A))$ be the original matrix $A$, that is,

$$
\operatorname{mat}(\operatorname{vec}(A))=A \text {. }
$$

Based on (2.1) and the fact that the matrix $X$ and $C$ are symmetric, problem (1.2) can similarly be rewritten as the following min-max problem:

$$
\min _{X \in S_{\Lambda}^{n}} \max _{Z \in \mathcal{B}} \operatorname{vec}(Z)^{T}(\operatorname{vec}(X)-\operatorname{vec}(C)),
$$

where

$$
\mathbb{B}=\left\{Z \in R^{n \times n}\left|Z=Z^{T}, \sum_{i=1}^{n} \sum_{j=1}^{n}\right| z_{i j} \mid \leq 1\right\} .
$$

Remark 2.1. Since $X$ and $C$ are both symmetric matrices, we can restrict the matrices in set $B$ to be symmetric.

Let

$$
\begin{gathered}
\Omega_{1}=\left\{x=\operatorname{vec}(X) \mid X \in S_{\Lambda}^{n}\right\}, \\
\Omega_{2}=\{z=\operatorname{vec}(Z) \mid Z \in B\} .
\end{gathered}
$$

Since $S_{\Lambda}^{n}$ and $\Pi$ are both convex sets, it is easy to prove that $\Omega_{1}$ and $\Omega_{2}$ are also convex sets.

Let $\left(X^{*}, Z^{*}\right) \in S_{\Lambda}^{n} \times B$ be any solution of $(2.4)$ and $x^{*}=\operatorname{vec}\left(X^{*}\right), z^{*}=\operatorname{vec}\left(Z^{*}\right)$. Then

$$
z^{T}\left(x^{*}-c\right) \leq z^{* T}\left(x^{*}-c\right) \leq z^{* T}(x-c), \quad \forall(x, z) \in \Omega,
$$

where $c=\operatorname{vec}(C)$ and $\Omega=\Omega_{1} \times \Omega_{2}$. Thus, $\left(x^{*}, z^{*}\right)$ is a solution of the following variational inequality: find $\left(x^{*}, z^{*}\right) \in \Omega$ such that

$$
\begin{gathered}
\left(x-x^{*}\right)^{T} z^{*} \geq 0, \quad \forall(x, z) \in \Omega . \\
\left(z-z^{*}\right)^{T}\left(-x^{*}+c\right) \geq 0, \quad
\end{gathered}
$$

For convenience of coming analysis, we rewrite the linear variational inequality (2.9) in the following compact form: find $u^{*} \in \Omega$ such that

$$
\left(u-u^{*}\right)^{T}\left(M u^{*}+q\right) \geq 0, \quad \forall u \in \Omega,
$$


where

$$
u^{*}=\left(\begin{array}{l}
x^{*} \\
z^{*}
\end{array}\right), \quad u=\left(\begin{array}{l}
x \\
z
\end{array}\right), \quad M=\left(\begin{array}{cc}
0 & I \\
-I & 0
\end{array}\right), \quad q=\left(\begin{array}{l}
0 \\
c
\end{array}\right)
$$

In the following, we denote the linear variational inequality (2.10)-(2.11) by $\operatorname{LVI}(\Omega, M, q)$.

Remark 2.2. Since $M$ is skew-symmetric, the linear variational inequality $\operatorname{LVI}(\Omega, M, q)$ is monotone.

\section{Projection and Contraction Method for Monotone LVIs}

In this section, we summarize some important concepts and preliminary results which are useful in the coming analysis.

\subsection{Projection Mapping}

Let $\Omega$ be a nonempty closed convex set of $R^{m}$. For a given $v \in R^{m}$, the projection of $v$ onto $\Omega$, denoted by $P_{\Omega}(v)$, is the unique solution of the following problem:

$$
\min _{u}\{\|u-v\| \mid u \in \Omega\},
$$

where $\|\cdot\|$ is the Euclidian norm. The projection under an Euclidean norm plays an important role in the proposed method. A basic property of the projection mapping on a closed convex set is

$$
\left(v-P_{\Omega}(v)\right)^{T}\left(u-P_{\Omega}(v)\right) \leq 0, \quad \forall v \in R^{m}, \forall u \in \Omega
$$

In many cases of practical applications, the closed convex set $\Omega$ has a simple structure, and the projection on $\Omega$ is easy to carry out. For example, let $e$ be a vector whose each element is 1 , and

$$
B_{\infty}=\left\{\xi \in R^{m} \mid\|\xi\|_{\infty} \leq 1\right\}
$$

Then the projection of a vector $d \in R^{m}$ on $\boldsymbol{B}_{\infty}$ can be obtained by

$$
P_{\mathbb{B}_{\infty}}(d)=\max \{-e, \min \{d, e\}\},
$$

where the min and max are component wise. 


\subsection{Preliminaries on Linear Variational Inequalities}

We denote the solution set of $\operatorname{LVI}(\Omega, M, q)(2.10)$ by $\Omega^{*}$ and assume that $\Omega^{*} \neq \emptyset$. Since the early work of Eaves [16], it is well known that the variational inequality $\operatorname{LVI}(\Omega, M, q)$ problem is equivalent to the following projection equation:

$$
u=P_{\Omega}[u-(M u+q)]
$$

In other words, to solve $\operatorname{LVI}(\Omega, M, q)$ is equivalent to finding a zero point of the continuous residue function

$$
e(u):=u-P_{\Omega}[u-(M u+q)]
$$

Hence,

$$
e(u)=0 \Longleftrightarrow u \in \Omega^{*}
$$

In the literature of variational inequalities, $\|e(u)\|$ is called the error bound of LVI. It quantitatively measures how much $u$ fails to be in $\Omega^{*}$.

\subsection{The Projection and Contraction Method}

Let $u^{*} \in \Omega^{*}$ be a solution. For any $u \in R^{m}$, because $P_{\Omega}[u-(M u+q)] \in \Omega$, it follows from (2.10) that

$$
\left(P_{\Omega}[u-(M u+q)]-u^{*}\right)^{T}\left(M u^{*}+q\right) \geq 0, \quad \forall u \in R^{m} .
$$

By setting $v=u-(M u+q)$ and $u=u^{*}$ in (3.2), we have

$$
\left(P_{\Omega}[u-(M u+q)]-u^{*}\right)^{T}\left\{u-(M u+q)-P_{\Omega}[u-(M u+q)]\right\} \geq 0, \quad \forall u \in R^{m} .
$$

Adding the above two inequalities, and using the notation of $e(u)$, we obtain

$$
\left\{\left(u-u^{*}\right)-e(u)\right\}^{T}\left\{e(u)-M\left(u-u^{*}\right)\right\} \geq 0, \quad \forall u \in R^{m} .
$$

For positive semi-definite (not necessary symmetric) matrix $M$, the following theorem follows from (3.10) directly.

Theorem 3.1 (Theorem 1 in [11]). For any $u^{*} \in \Omega^{*}$, we have

$$
\left(u-u^{*}\right)^{T} d(u) \geq\|e(u)\|^{2}, \quad \forall u \in R^{m},
$$

where

$$
d(u)=M^{T} e(u)+e(u) .
$$


For $u \in \Omega \backslash \Omega^{*}$, it follows from (3.11)-(3.12) that $-d(u)$ is a descent direction of the unknown function $(1 / 2)\left\|u-u^{*}\right\|^{2}$. Some practical PC methods for LVI based on direction $d(u)$ are given in [11].

Algorithm 3.2 (Projection and Contraction Method for LVI Problem (2.10)). Given $u^{0} \in \Omega$. for $k=0,1 \ldots$, if $u^{k} \notin \Omega^{*}$, then do the following

$$
u^{k+1}=u^{k}-\gamma \alpha\left(u^{k}\right) d\left(u^{k}\right)
$$

where $\gamma \in(0,2), d(u)$ is defined in (3.12), and

$$
\alpha(u)=\frac{\|e(u)\|^{2}}{\|d(u)\|^{2}} .
$$

Remark 3.3. In fact, $\gamma$ is a relaxation factor and it is recommended to be taken from $[1,2)$. In practical computation, usually we take $\gamma \in[1.2,1.9]$. The method was first mentioned in [11]. Among the PC methods for asymmetric $\operatorname{LVI}(\Omega, M, q)[10-13]$, this method makes just one projection in each iteration.

For completeness sake, we include the theorem for LVI (2.10) and its proofs.

Theorem 3.4 (Theorem 2 in [11]). The method (3.13)-(3.14) produces a sequence $\left\{u^{k}\right\}$, which satisfies

$$
\left\|u^{k+1}-u^{*}\right\|^{2} \leq\left\|u^{k}-u^{*}\right\|^{2}-\gamma(2-\gamma) \alpha\left(u^{k}\right)\left\|e\left(u^{k}\right)\right\|^{2}
$$

Proof. It follows from (3.11) and (3.14) that

$$
\begin{aligned}
\left\|u^{k+1}-u^{*}\right\|^{2} & =\left\|u^{k}-u^{*}-\gamma \alpha\left(u^{k}\right) d\left(u^{k}\right)\right\|^{2} \\
& =\left\|u^{k}-u^{*}\right\|^{2}-2 \gamma \alpha\left(u^{k}\right)\left(u^{k}-u^{*}\right)^{T} d\left(u^{k}\right)+\gamma^{2} \alpha^{2}\left(u^{k}\right)\left\|d\left(u^{k}\right)\right\|^{2} \\
& \leq\left\|u^{k}-u^{*}\right\|^{2}-2 \gamma \alpha\left(u^{k}\right)\left\|e\left(u^{k}\right)\right\|^{2}+\gamma^{2} \alpha^{2}\left(u^{k}\right)\left\|d\left(u^{k}\right)\right\|^{2} \\
& =\left\|u^{k}-u^{*}\right\|^{2}-\gamma(2-\gamma) \alpha\left(u^{k}\right)\left\|e\left(u^{k}\right)\right\|^{2}
\end{aligned}
$$

Thus the theorem is proved.

The method used in this paper is called projection and contraction method because it makes projections in each iteration and the generated sequence is Fejér monotone with respect to the solution set.

For skew-symmetric $M$ in LVI (2.10), it is easy to prove that $\alpha(u) \equiv 1 / 2$. Thus, the contraction inequality (3.15) can be simplified to

$$
\left\|u^{k+1}-u^{*}\right\|^{2} \leq\left\|u^{k}-u^{*}\right\|^{2}-\frac{\gamma(2-r)}{2}\left\|e\left(u^{k}\right)\right\|^{2} .
$$


Following (3.17), the convergence results of the sequence $\left\{u_{k}\right\}$ can be found in [11] or be proved similarly to that in [17].

Since the above inequality is true for all $u^{*} \in \Omega^{*}$, we have

$$
\operatorname{dist}^{2}\left(u^{k+1}, \Omega^{*}\right) \leq \operatorname{dist}^{2}\left(u^{k}, \Omega^{*}\right)-\frac{\gamma(2-\gamma)}{2}\left\|e\left(u^{k}\right)\right\|^{2}
$$

where

$$
\operatorname{dist}\left(u, \Omega^{*}\right)=\min \left\{\left\|u-u^{*}\right\| \mid u^{*} \in \Omega^{*}\right\} .
$$

The above inequality states that we get a "great" profit from the $k$ th iteration, if $\left\|e\left(u^{k}\right)\right\|$ is not too small; conversely, if we get a very small profit from the $k$ th iteration, then $\left\|e\left(u^{k}\right)\right\|$ is already very small and $u^{k}$ is a "sufficiently good" approximation of a $u^{*} \in \Omega^{*}$.

\section{Implementing Details of Algorithm 3.2 for Solving Problem (1.2)}

We use the Algorithm 3.2 to solve the linear variational inequality (2.10) arising from problem (1.2). For a given $u=(x, z) \in \Omega$, the process of computing a new iterate is listed as follows:

$$
\begin{gathered}
M u+q=\left(\begin{array}{c}
z \\
c-x
\end{array}\right), \\
e(u)=\left(\begin{array}{c}
e_{x}(u) \\
e_{z}(u)
\end{array}\right)=\left(\begin{array}{c}
x-P_{\Omega_{1}}[x-z] \\
z-P_{\Omega_{2}}[z+(x-c)]
\end{array}\right), \\
d(u)=\left(\begin{array}{c}
d_{x}(u) \\
d_{z}(u)
\end{array}\right)=M^{T} e(u)+e(u)=\left(\begin{array}{c}
e_{x}(u)-e_{z}(u) \\
e_{x}(u)+e_{z}(u)
\end{array}\right), \\
u^{\text {new }}=\left(\begin{array}{c}
x^{\text {new }} \\
z^{\text {new }}
\end{array}\right)=\left(\begin{array}{c}
x-\frac{\gamma d_{x}(u)}{2} \\
z-\frac{r d_{z}(u)}{2}
\end{array}\right), \quad r \in(0,2) .
\end{gathered}
$$

The key operations here are to compute $P_{\Omega_{1}}[x-z]$ and $P_{\Omega_{2}}[z+(x-c)]$, where $\operatorname{mat}(x)$, mat $(z)$, and $\operatorname{mat}(z+(x-c))$ are symmetric matrices. In the following, we first focus on the computing method of $P_{\Omega_{1}}[v]$, where mat $(v)$ is a symmetric matrix.

Since $\operatorname{mat}(v)$ is a symmetric matrix, we have

$$
P_{\Omega_{1}}[v]=\arg \min _{x}\left\{\|x-v\|^{2} \mid x \in \Omega_{1}\right\}=\operatorname{vec}\left(\arg \min _{X}\left\{\|X-V\|_{F}^{2} \mid X \in S_{\Lambda}^{n}\right\}\right),
$$

where $V=\operatorname{mat}(v)$. It is known that the optimal solution of problem

$$
\min \left\{\|X-V\|_{F}^{2} \mid X \in S_{\Lambda}^{n}\right\}
$$


is given by

$$
Q \tilde{\Lambda} Q^{T}
$$

where

$$
\begin{gathered}
V Q=Q \Lambda, \quad \Lambda=\operatorname{diag}\left(\lambda_{11}, \ldots, \lambda_{n n}\right), \\
\tilde{\Lambda}=\operatorname{diag}\left(\tilde{\lambda}_{11}, \ldots, \tilde{\lambda}_{n n}\right), \quad \tilde{\lambda}_{i i}=\min \left\{\max \left\{\lambda_{i i}, \lambda_{\min }\right\}, \lambda_{\max }\right\},
\end{gathered}
$$

and $\|\cdot\|_{F}$ is the Frobenius norm. Thus, we have

$$
P_{\Omega_{1}}[v]=\operatorname{vec}\left(Q \tilde{\Lambda} Q^{T}\right)
$$

Now we move to consider the technique of computing $P_{\Omega_{2}}[v]$, where mat $(v)$ is a symmetric matrix.

Lemma 4.1. If $\operatorname{mat}(v)$ is a symmetric matrix and $x^{*}$ is the solution of the problem

$$
\min _{x}\left\{\|x-v\|\left|\sum_{i=1}^{m}\right| x_{i} \mid \leq 1\right\}
$$

then we have

$$
\operatorname{mat}\left(x^{*}\right)=\operatorname{mat}\left(x^{*}\right)^{T}
$$

Proof. Since

$$
\arg \min _{x}\left\{\|x-v\|\left|\sum_{i=1}^{m}\right| x_{i} \mid \leq 1\right\}=\operatorname{vec}\left(\arg \min _{X}\left\{\|X-V\|_{F}\left|\sum_{i=1}^{n} \sum_{j=1}^{n}\right| x_{i j} \mid \leq 1\right\}\right)
$$

and $V=V^{T}$, where $V=\operatorname{mat}(v)$, we have that if $x^{*}$ is the solution of problem (4.7), then $\operatorname{vec}\left(\operatorname{mat}\left(x^{*}\right)^{T}\right)$ is also the solution of problem (4.7). As it is known that the solution of problem (4.7) is unique. Thus, $x^{*}=\operatorname{vec}\left(\operatorname{mat}\left(x^{*}\right)^{T}\right)$, and the proof is complete.

Lemma 4.2. If $x^{*}$ is the solution of problem

$$
\min _{x}\left\{\|x-\operatorname{sign}(v) \circ v\| \mid \sum_{i=1}^{m} x_{i} \leq 1, x \geq 0\right\}
$$

where $\operatorname{sign}(v)=\left(\operatorname{sign}\left(v_{1}\right), \operatorname{sign}\left(v_{2}\right), \ldots, \operatorname{sign}\left(v_{m}\right)\right)^{T}, \operatorname{sign}\left(v_{i}\right)$ is the sign of real number $v_{i}$, and

$$
\operatorname{sign}(v) \circ v=\left(\operatorname{sign}\left(v_{1}\right) v_{1}, \operatorname{sign}\left(v_{2}\right) v_{2}, \ldots, \operatorname{sign}\left(v_{m}\right) v_{m}\right)^{T}=|v|,
$$


then $\operatorname{sign}(v) \circ x^{*}$ is the solution of the problem

$$
\min _{x}\left\{\|x-v\|\left|\sum_{i=1}^{m}\right| x_{i} \mid \leq 1\right\} .
$$

Proof. The result follows from

$$
\begin{gathered}
\|x-v\|=\|\operatorname{sign}(v) \circ(x-v)\|, \\
\sum_{i=1}^{m}\left|\operatorname{sign}\left(v_{i}\right) x_{i}\right|=\sum_{i=1}^{m}\left|x_{i}\right| \leq 1 .
\end{gathered}
$$

Lemma 4.3. Let $v \geq 0, T$ be a permutation transformation sorting the components of $v$ in descending order, that is, the components of $\bar{v}=T v$ are in descending order. Further, suppose that $\bar{x}^{*}$ is the solution of the problem

$$
\min _{x}\left\{\|x-\bar{v}\| \mid \sum_{i=1}^{m} x_{i} \leq 1\right\}
$$

then $x^{*}=T^{-1} \bar{x}^{*}$ is the solution of the problem

$$
\min _{x}\left\{\|x-v\| \mid \sum_{i=1}^{m} x_{i} \leq 1\right\} .
$$

Proof. Since $T$ is a permutation transformation, we have that

$$
T^{-1}=T,
$$

and the optimal values of objective function of problem (4.14) and (4.15) are equal.

Note that

$$
\begin{gathered}
\left\|x^{*}-v\right\|=\left\|T\left(x^{*}-v\right)\right\|=\left\|\bar{x}^{*}-\bar{v}\right\|, \\
\sum_{i=1}^{m} x_{i}^{*}=\sum_{i=1}^{m} \bar{x}_{i}^{*} \leq 1 .
\end{gathered}
$$

Thus, $x^{*}$ is the optimal solution of problem (4.15). And the proof is complete. 
$\operatorname{Remark}$ 4.4. Suppose that $\operatorname{mat}(v) \in R^{n \times n}$ is a symmetric matrix for a given $v \in R^{m}$. Let $\widetilde{v}=$ $T(\operatorname{sign}(v) \circ v)$, where $T$ is a permutation transformation sorting the components of $\operatorname{sign}(v) \circ v$ in descending order. Lemmas 4.1-4.3 show that if $\tilde{x}^{*}$ is the solution of the following problem

$$
\min _{x}\left\{\frac{1}{2}\|x-\tilde{v}\|^{2} \mid \sum_{i=1}^{m} x_{i} \leq 1, x \geq 0\right\},
$$

then

$$
P_{\Omega_{2}}[v]=\operatorname{sign}(v) \circ\left(T \tilde{x}^{*}\right)
$$

Hence, to solve the problem (4.18) is a key work to obtain the projection $P_{\Omega_{2}}[v]$. Let $e=(1,1, \ldots, 1)^{T} \in R^{m}$, then problem (4.18) can be rewritten as

$$
\min _{x}\left\{\frac{1}{2}\|x-\widetilde{v}\|^{2} \mid e^{T} x \leq 1, x \geq 0\right\}
$$

The Lagrangian function for the constrained optimal problem (4.20) is defined as

$$
L(x, y, w)=\frac{1}{2}\|x-\widetilde{v}\|^{2}-y\left(1-e^{T} x\right)-w^{T} x,
$$

where scale $y$ and vector $w \in R^{m}$ are the Lagrange multipliers corresponding to inequalities $e^{T} x \leq 1$ and $x \geq 0$, respectively. By KKT condition, we have

$$
\begin{gathered}
x-\tilde{v}+y e-w=0, \\
y \geq 0, \quad 1-e^{T} x \geq 0, \quad y\left(1-e^{T} x\right)=0, \\
w \geq 0, \quad x \geq 0, \quad w^{T} x=0,
\end{gathered}
$$

that is,

$$
\begin{gathered}
y \geq 0, \quad 1-e^{T} x \geq 0, \quad y\left(1-e^{T} x\right)=0, \\
x \geq 0, \quad x+y e-\tilde{v} \geq 0, \quad x^{T}(x+y e-\tilde{v})=0 .
\end{gathered}
$$

It is easy to check that if $e^{T} \widetilde{v} \leq 1$, then $\left(\tilde{x}^{*}, \tilde{y}^{*}\right)=(\widetilde{v}, 0)$ is the solution of problem (4.23). Now we assume that $e^{T} \tilde{v}>1$. In this case, let

$$
\Delta v=\left(\widetilde{v}_{1}-\widetilde{v}_{2}, \widetilde{v}_{2}-\widetilde{v}_{3}, \ldots, \widetilde{v}_{m-1}-\widetilde{v}_{m}, \widetilde{v}_{m}\right)^{T}
$$


Note that $\Delta v \geq 0$ and

$$
\sum_{i=1}^{m} i \times \Delta v_{i}=\sum_{i=1}^{m} \widetilde{v}_{i}>1
$$

Thus, there exists a least-integer $K$ such that

$$
\sum_{i=1}^{K} i \times \Delta v_{i} \geq 1
$$

Since

$$
\sum_{i=1}^{K} \widetilde{v}_{i}= \begin{cases}\sum_{i=1}^{K} i \times \Delta v_{i}+K \times \widetilde{v}_{K+1}, & 1 \leq K<m \\ \sum_{i=1}^{K} i \times \Delta v_{i}, & K=m\end{cases}
$$

we have that

$$
\sum_{i=1}^{K} \tilde{v}_{i} \geq 1
$$

Let

$$
\begin{gathered}
\tilde{y}^{*}=\frac{1}{K}\left(\sum_{i=1}^{K} \widetilde{v}_{i}-1\right), \\
\tilde{x}^{*}=\left(\widetilde{v}_{1}-\tilde{y}^{*}, \widetilde{v}_{2}-\tilde{y}^{*}, \ldots, \widetilde{v}_{K}-\tilde{y}^{*}, 0, \ldots, 0\right)^{T} \in R^{m} .
\end{gathered}
$$

Theorem 4.5. Let $\tilde{x}^{*}$ and $\tilde{y}^{*}$ be given by (4.30) and (4.29), respectively, then $\left(\tilde{x}^{*}, \tilde{y}^{*}\right)$ is the solution of problem (4.23), and thus

$$
P_{\Omega_{2}}[v]=\operatorname{sign}(v) \circ\left(T \tilde{x}^{*}\right)
$$

Proof. It follows from (4.26), (4.27), and (4.29) that

$$
\begin{aligned}
\tilde{y}^{*} & =\frac{1}{K}\left(\sum_{i=1}^{K} \widetilde{v}_{i}-1\right) \geq \frac{1}{K} \sum_{i=1}^{K}\left(\widetilde{v}_{i}-i \times \Delta v_{i}\right) \\
& = \begin{cases}\tilde{v}_{K+1}, & 1 \leq K<m, \\
0, & K=m,\end{cases} \\
\tilde{y}^{*} & =\frac{1}{K}\left(\sum_{i=1}^{K} \widetilde{v}_{i}-1\right) \leq \frac{1}{K}\left(\sum_{i=1}^{K} \widetilde{v}_{i}-\sum_{i=1}^{K-1} i \times \Delta v_{i}\right)
\end{aligned}
$$




$$
\begin{aligned}
& =\frac{1}{K}\left(\sum_{i=1}^{K-1} \widetilde{v}_{i}-\sum_{i=1}^{K-1} i \times \Delta v_{i}+\widetilde{v}_{K}\right) \\
& =\widetilde{v}_{K} .
\end{aligned}
$$

Following from (4.32) it is easy to check that $\left(\tilde{x}^{*}, \tilde{y}^{*}\right)$ is a solution of problem (4.23). Note that problem (4.18) is convex, thus $\left(\tilde{x}^{*}, \tilde{y}^{*}\right)$ is the solution of problem (4.18). Further, according to Remark 4.4, we have

$$
P_{\Omega_{2}}[v]=\operatorname{sign}(v) \circ\left(T \tilde{x}^{*}\right)
$$

The proof is complete.

Remark 4.6. Note that if $\tilde{X}^{*}$ is the solution of problem

$$
\min \left\{\left\|X-\frac{1}{\beta} C\right\|_{\text {inf }} \mid X=\left(x_{i j}\right) \in \widetilde{S}_{\Lambda}^{n}\right\}
$$

where $\beta>0$ is a given scalar, and

$$
\widetilde{S}_{\Lambda}^{n}=\left\{H \in R^{n \times n} \mid H^{T}=H, \frac{\lambda_{\min }}{\beta} I \preceq H \preceq \frac{\lambda_{\max }}{\beta} I\right\},
$$

then $X^{*}=\beta \tilde{X}^{*}$ is the solution of problem (1.2). Thus, we can find the solution of problem (1.2) by solving problem (4.34).

Let

$$
\widetilde{\Omega}_{1}=\left\{x=\operatorname{vec}(X) \mid X \in \widetilde{S}_{\Lambda}^{n}\right\} .
$$

Now, we are in the stage to describe the implementing process of Algorithm 3.2 for problem (1.2) in detail.

Algorithm 4.7 (projection and contraction method for problem (1.2)).

Step 1 (Initialization). Let $C=\left(c_{i j}\right) \in R^{n \times n}$ be a given symmetric matrix, $\lambda_{\min }, \lambda_{\max }, \beta>$ 0 be given scalars. Choose arbitrarily an initial point $x^{0}=\operatorname{vec}\left(X^{0}\right), z^{0}=\operatorname{vec}\left(Z^{0}\right)$, and $u^{0}=$ $\left(\begin{array}{c}x^{0} \\ z^{0}\end{array}\right)$, where $X^{0} \in \tilde{\Omega}_{1}, Z^{0} \in \Omega_{2}, \tilde{\Omega}_{1}$, and $\Omega_{2}$ are defined by (4.36) and (2.7), respectively. Let $\gamma \in(0,2), k=0$, and $\varepsilon>0$ be a prespecified tolerance.

Step 2 (Computation). Compute $P_{\widetilde{\Omega}_{1}}\left[x^{k}-z^{k}\right]$ and $P_{\Omega_{2}}\left[z^{k}+\left(x^{k}-c\right)\right]$ by using (4.6) and (4.31), respectively.

Let

$$
\begin{aligned}
& e_{x}\left(u^{k}\right)=x^{k}-P_{\tilde{\Omega}_{1}}\left[x^{k}-z^{k}\right], \\
& e_{z}\left(u^{k}\right)=z^{k}-P_{\Omega_{2}}\left[z^{k}+\left(x^{k}-c\right)\right],
\end{aligned}
$$


Table 1: Numerical results of example 1.

\begin{tabular}{lcccccc}
\hline$n$ & $\lambda_{1}$ & $\lambda_{n}$ & $k$ & CPU time $(\mathrm{s})$ & $\|e(u)\|_{\infty}$ & $\|\operatorname{vec}(X-C)\|_{\infty} /\|\operatorname{vec}(C)\|_{\infty}$ \\
\hline 10 & 0.2947 & 2.2687 & 55 & 0.06 & $9.2699 \times 10^{-8}$ & $1.9768 \times 10^{-7}$ \\
20 & -0.5849 & 2.8134 & 58 & 0.17 & $7.3282 \times 10^{-8}$ & $2.7002 \times 10^{-7}$ \\
30 & -0.9467 & 2.9938 & 65 & 0.35 & $7.5651 \times 10^{-8}$ & $2.8008 \times 10^{-7}$ \\
40 & -1.2405 & 3.4024 & 75 & 0.49 & $9.6325 \times 10^{-8}$ & $5.0350 \times 10^{-7}$ \\
50 & -1.7593 & 3.7925 & 89 & 0.67 & $8.5592 \times 10^{-8}$ & $3.9993 \times 10^{-7}$ \\
60 & -2.0733 & 4.1648 & 106 & 1.29 & $8.9517 \times 10^{-8}$ & $6.1197 \times 10^{-7}$ \\
70 & -2.3450 & 4.3352 & 121 & 2.00 & $9.5952 \times 10^{-8}$ & $6.6696 \times 10^{-7}$ \\
80 & -2.5817 & 4.5194 & 140 & 3.56 & $8.3927 \times 10^{-8}$ & $3.7658 \times 10^{-7}$ \\
90 & -2.8345 & 4.7512 & 160 & 5.23 & $8.3227 \times 10^{-8}$ & $4.8914 \times 10^{-7}$ \\
100 & -2.9193 & 5.1780 & 181 & 7.43 & $9.3473 \times 10^{-8}$ & $6.2160 \times 10^{-7}$ \\
150 & -4.1850 & 5.7006 & 302 & 35.90 & $7.4435 \times 10^{-8}$ & $6.1552 \times 10^{-7}$ \\
200 & -4.6721 & 6.7545 & 446 & 119.07 & $7.8171 \times 10^{-8}$ & $8.4093 \times 10^{-7}$ \\
\hline
\end{tabular}

Table 2: Numerical results of example 2.

\begin{tabular}{lcccccc}
\hline$n$ & $\lambda_{1}$ & $\lambda_{n}$ & $k$ & CPU time $(\mathrm{s})$ & $\|e(u)\|_{\infty}$ & $\|\operatorname{vec}(X-C)\|_{\infty} /\|\operatorname{vec}(C)\|_{\infty}$ \\
\hline 10 & 0.0090 & 9.0559 & 74 & 0.08 & $9.9036 \times 10^{-8}$ & $4.2676 \times 10^{-9}$ \\
20 & 0.0048 & 19.4004 & 107 & 0.30 & $9.4778 \times 10^{-8}$ & $4.2266 \times 10^{-7}$ \\
30 & 0.0184 & 33.6477 & 7617 & 17.98 & $9.9976 \times 10^{-8}$ & $7.0348 \times 10^{-2}$ \\
40 & 0.0004 & 46.4279 & 162 & 0.76 & $9.9938 \times 10^{-8}$ & $1.2216 \times 10^{-1}$ \\
50 & 0.0045 & 55.7072 & 192 & 1.46 & $9.4131 \times 10^{-8}$ & $1.8517 \times 10^{-1}$ \\
60 & 0.0020 & 73.8863 & 1259 & 15.18 & $9.9708 \times 10^{-8}$ & $2.4254 \times 10^{-1}$ \\
70 & 0.0084 & 86.8784 & 245 & 4.28 & $8.9912 \times 10^{-8}$ & $3.3916 \times 10^{-1}$ \\
80 & 0.0021 & 100.3238 & 614 & 14.13 & $9.0897 \times 10^{-8}$ & $3.8534 \times 10^{-1}$ \\
90 & 0.0002 & 108.6724 & 384 & 10.38 & $7.7490 \times 10^{-8}$ & $4.7801 \times 10^{-1}$ \\
100 & 0.0011 & 130.7923 & 326 & 9.03 & $9.0180 \times 10^{-8}$ & $5.2592 \times 10^{-1}$ \\
150 & 0.0001 & 195.6736 & 2214 & 98.28 & $7.0598 \times 10^{-8}$ & $6.6452 \times 10^{-1}$ \\
200 & 0.0013 & 261.9417 & 1582 & 165.29 & $7.1460 \times 10^{-8}$ & $7.4489 \times 10^{-1}$ \\
\hline
\end{tabular}

$$
\begin{aligned}
& d_{x}\left(u^{k}\right)=e_{x}\left(u^{k}\right)-e_{z}\left(u^{k}\right), \\
& d_{z}\left(u^{k}\right)=e_{x}\left(u^{k}\right)+e_{z}\left(u^{k}\right),
\end{aligned}
$$

where $u^{k}=\left(\begin{array}{c}x^{k} \\ z^{k}\end{array}\right)$.

Step 3. (Verification). If $\left\|e\left(u^{k}\right)\right\|_{\infty}<\varepsilon$, then stop and output the approximate solution $X=\beta \operatorname{mat}\left(x^{k}\right)$, where $e\left(u^{k}\right)=\left(\begin{array}{c}e_{x}\left(u^{k}\right) \\ e_{z}\left(u^{k}\right)\end{array}\right)$.

Step 4. (Iteration).

$$
\begin{aligned}
& x^{k+1}=x^{k}-\gamma d_{x}\left(u^{k}\right) / 2, \\
& z^{k+1}=z^{k}-\gamma d_{z}\left(u^{k}\right) / 2, \\
& k:=k+1, \text { goto Step 2. }
\end{aligned}
$$

\section{Numerical Experiments}

In this section, some examples are provided to illustrate the performance of Algorithm 4.7 for solving problem (1.2). In the following illustrative examples, the computer program for 
implementing Algorithm 4.7 is coded in Matlab and the program runs on IBM notebook (R51).

Example 5.1. Consider problem (1.2) with $C=\left(\widetilde{C}+\widetilde{C}^{T}\right) / 2+\operatorname{eye}(n), \lambda_{\min }=-50$, and $\lambda_{\max }=50$, where $\widetilde{C}=\operatorname{rand}(n)-0.5$, rand and eye are both the Matlab functions, and $n$ is the size of problem (1.2). Let $\gamma=1.5, \varepsilon=1 \times 10^{-7}$, then we have

$$
\beta=\sqrt{n}\|\operatorname{vec}(C)\|_{\infty}, \quad x_{0}=\operatorname{vec}\left(\frac{1}{2}\left(\tilde{\lambda}_{1}+\tilde{\lambda}_{2}\right) \times \operatorname{eye}(n)\right), \quad z_{0}=\operatorname{vec}(\operatorname{zeros}(n)),
$$

where zeros is also the Matlab function, $\tilde{\lambda}_{1}=\max \left\{\lambda_{1} / \beta, \lambda_{\min } / \beta\right\}, \tilde{\lambda}_{2}=\min \left\{\lambda_{n} / \beta, \lambda_{\max } / \beta\right\}$, and $\lambda_{1}, \lambda_{n}$ are the smallest and the largest eigenvalue of matrix $C$, respectively. Table 1 reports the numerical results of Example 5.1 solved by Algorithm 4.7, where $k$ is the number of iterations, the unit of time is second, and $X$ is the approximate solution of problem (1.2) obtained by Algorithm 4.7.

Example 5.2. Consider problem (1.2) with $C=\widetilde{C} \widetilde{C}^{T}, \lambda_{\min }=0$, and $\lambda_{\max }=20$, where $\widetilde{C}=$ $2 \times \operatorname{rand}(n)-1$. In this test example, let $\gamma, \varepsilon$ be same as that in Example 5.1, $\beta, x_{0}$, and $z_{0}$ be also given according to (5.1). Table 2 reports the numerical results of Example 5.2 and shows the numerical performance of Algorithm 4.7 for solving problem (1.2).

\section{Conclusions}

In this paper, a relationship between the matrix nearness problem and the linear variational inequality has been built. The matrix nearness problem considered in this paper can be solved by applying an algorithm for the related linear variational inequality. Based on this point, a projection and contraction method is presented for solving the matrix nearness problem, and the implementing details are introduced in this paper. Numerical experiments show that the method suggested in this paper has a good performance, and the method can be improved by setting the parameters in Algorithm 4.7 properly. Thus, further studying of the effect of the parameters in Algorithm 4.7 maybe a very interesting work.

\section{Acknowledgments}

This research is financially supported by a research grant from the Research Grant Council of China (Project no. 10971095).

\section{References}

[1] S. Boyd and L. Vandenberghe, Convex Optimization, Cambridge University Press, 2004.

[2] N. J. Higham, "Matrix nearness problems and applications," in Applications of Matrix Theory, M. Gover and S. Barnett, Eds., pp. 1-27, Oxford University Press, Oxford, UK, 1989.

[3] S. Boyd and L. Xiao, "Least-squares covariance matrix adjustment," SIAM Journal on Matrix Analysis and Applications, vol. 27, no. 2, pp. 532-546, 2005.

[4] N. J. Higham, "Computing a nearest symmetric positive semidefinite matrix," Linear Algebra and its Applications, vol. 103, pp. 103-118, 1988.

[5] N. J. Higham, "Computing the nearest correlation matrix-a problem from finance," IMA Journal of Numerical Analysis, vol. 22, no. 3, pp. 329-343, 2002. 
[6] G. L. Xue and Y. Y. Ye, "An efficient algorithm for minimizing a sum of Euclidean norms with applications," SIAM Journal on Optimization, vol. 7, no. 4, pp. 1017-1036, 1997.

[7] G. L. Xue and Y. Y. Ye, "An efficient algorithm for minimizing a sum of $p$-norms," SIAM Journal on Optimization, vol. 10, no. 2, pp. 551-579, 2000.

[8] J. Yang and Y. Zhang, "Alternating direction algorithms for $\ell_{1}$-problems in compressive sensing," SIAM Journal on Scientific Computing, vol. 33, no. 1, pp. 250-278, 2011.

[9] I. S. Dhillon and J. A. Tropp, "Matrix nearness problems with Bregman divergences," SIAM Journal on Matrix Analysis and Applications, vol. 29, no. 4, pp. 1120-1146, 2007.

[10] B. S. He, "A projection and contraction method for a class of linear complementarity problems and its application in convex quadratic programming," Applied Mathematics and Optimization, vol. 25, no. 3, pp. 247-262, 1992.

[11] B. S. He, "A new method for a class of linear variational inequalities," Mathematical Programming, vol. 66, no. 2, pp. 137-144, 1994.

[12] B. S. He, "Solving a class of linear projection equations," Numerische Mathematik, vol. 68, no. 1, pp. 71-80, 1994.

[13] B. S. He, "A modified projection and contraction method for a class of linear complementarity problems," Journal of Computational Mathematics, vol. 14, no. 1, pp. 54-63, 1996.

[14] B. He, "A class of projection and contraction methods for monotone variational inequalities," Applied Mathematics and Optimization, vol. 35, no. 1, pp. 69-76, 1997.

[15] M. H. Xu and T. Wu, "A class of linearized proximal alternating direction methods," Journal of Optimization Theory and Applications, vol. 151, no. 2, pp. 321-337, 2011.

[16] B. C. Eaves, "On the basic theorem of complementarity," Mathematical Programming, vol. 1, no. 1, pp. 68-75, 1971.

[17] M. H. Xu, J. L. Jiang, B. Li, and B. Xu, "An improved prediction-correction method for monotone variational inequalities with separable operators," Computers $\mathcal{E}$ Mathematics with Applications, vol. 59, no. 6, pp. 2074-2086, 2010. 


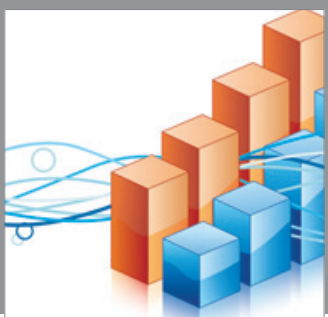

Advances in

Operations Research

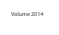

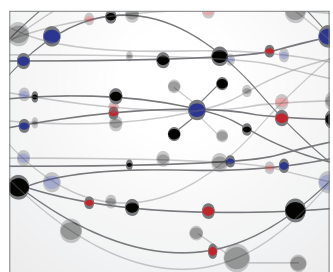

\section{The Scientific} World Journal
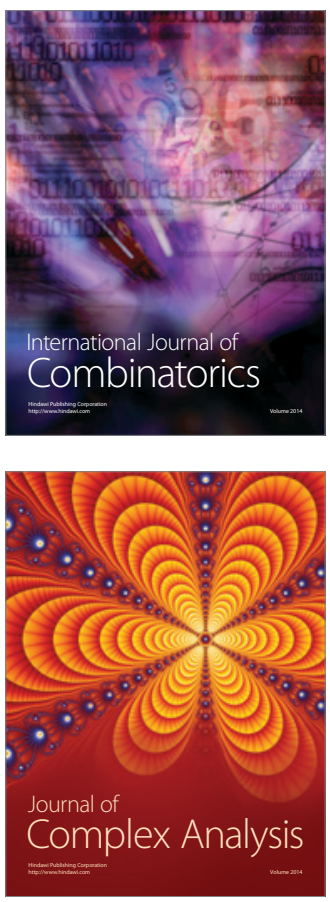

International Journal of

Mathematics and

Mathematical

Sciences
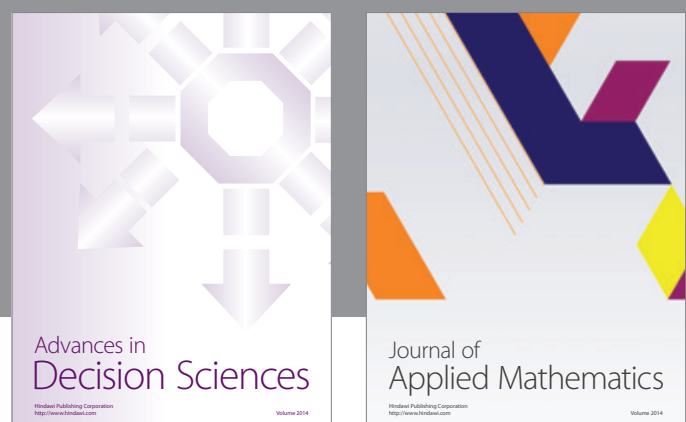

Journal of

Applied Mathematics
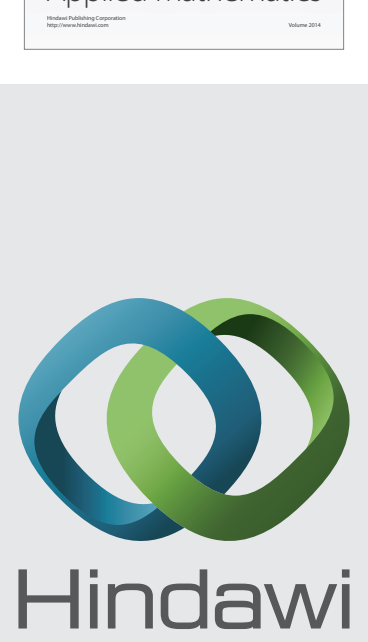

Submit your manuscripts at http://www.hindawi.com
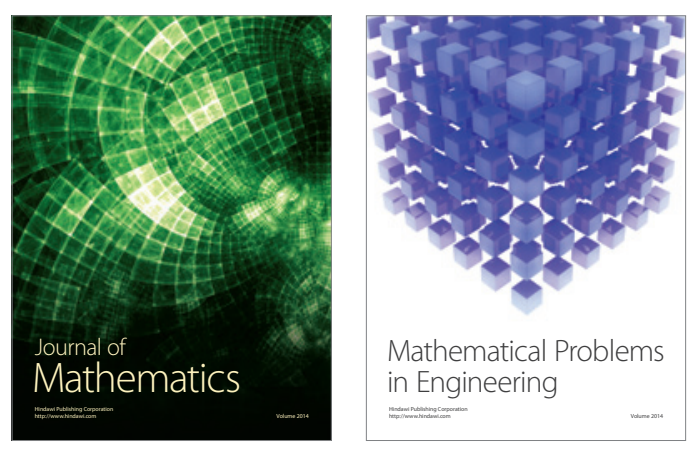

Mathematical Problems in Engineering
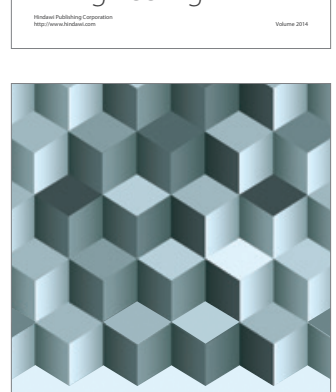

Journal of

Function Spaces
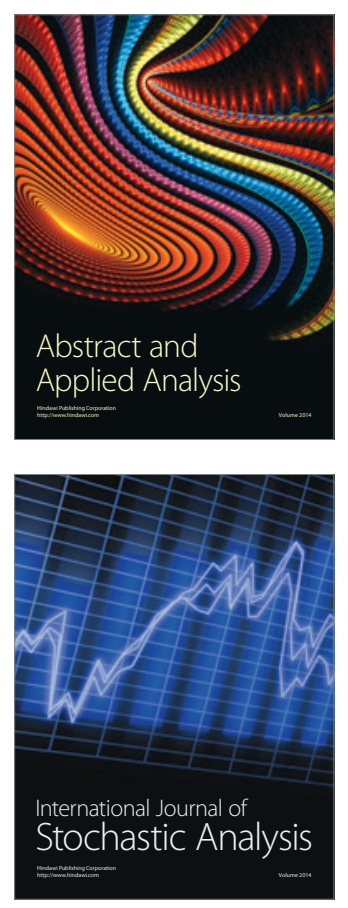

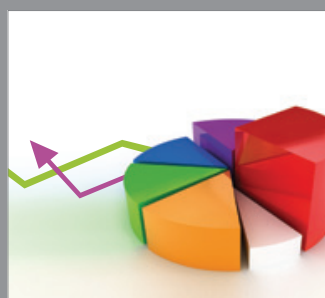

ournal of

Probability and Statistics

Promensencen
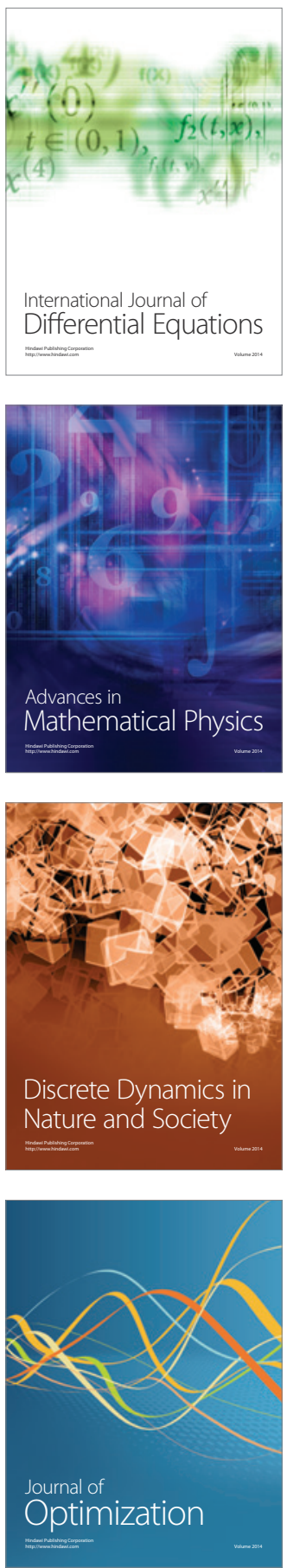